\title{
Kompetenzbasierter Lernzielkatalog „Rehabilitation, Physikalische Medizin, Naturheilverfahren“ (RPMN) - revidierte Fassung
}

Gemeinsame Empfehlung der Deutschen Gesellschaft für

Rehabilitationswissenschaften e.V. (DGRW) und der Deutschen

Gesellschaft für Physikalische Medizin und Rehabilitation e.V. (DGPMR)

\section{Competence Based Catalogue of Learning Objectives for Rehabilitation, Physical Medicine, Naturopathic Treatment - Revised Version}

Joint Recommendations of the German Society of Rehabilitation Science and the German Society of Physical Medicine and Rehabilitation

Autoren

Wilfried Mau*1, Max Emanuel Liebl ${ }^{* 2}$, Ruth Deck³, Uwe Lange4, Anett Reißhauer², Susanne Roswitha Schwarzkopf ${ }^{5}$, Ulrich Christian Smolenski ${ }^{6}$, Susanne Walter ${ }^{7}$, Christoph Gutenbrunner ${ }^{8}$

(für die Kommissionen Aus-, Fort- und Weiterbildung der DGRW\# und DGPMR ${ }^{+}$)

Institute

1 Institut für Rehabilitationsmedizin, Medizinische Fakultät der Martin-Luther-Universität Halle-Wittenberg

2 Charité - Universitätsmedizin Berlin, Physikalische Medizin und Rehabilitation, Berlin

3 Institut für Sozialmedizin und Epidemiologie, Universität zu Lübeck

4 Kerckhoff-Klinik, Bad Nauheim; Professur für Internistische Rheumatologie, Osteologie, Physikalische Medizin, Justus-Liebig-Universität Gießen

5 Klinik und Institut für Physikalische und Rehabilitative Medizin, Klinikum Nürnberg

6 Institut für Physiotherapie, Universitätsklinikum Jena

7 Sektion Versorgungsforschung und Rehabilitationsforschung, Institut für Medizinische Biometrie und Statistik, Universitätsklinikum Freiburg

8 Klinik für Rehabilitationsmedizin, Medizinische Hochschule Hannover

Schlüsselwörter Rehabilitation, Physikalische Medizin, Naturheilverfahren, Medizinstudium, Lernziele

\section{Key words}

Rehabilitation, Physical Medicine, Naturopathic Treatment, undergraduate medical education, learning objectives
Bibliografie

DOI https://doi.org/10.1055/s-0043-119760

Rehabilitation 2017; 56: 397-411

(c) Georg Thieme Verlag KG Stuttgart · New York ISSN 0034-3536

Korrespondenzadresse

Prof. Dr. med. Wilfried Mau

Institut für Rehabilitationsmedizin

Medizinische Fakultät der Martin-Luther-Universität

Halle-Wittenberg

Magdeburger Straße 8, 06097 Halle (Saale)

wilfried.mau@medizin.uni-halle.de

\footnotetext{
* W. Mau und M. Liebl haben das Manuskript zu gleichen Teilen erstellt. \# Mitglieder der Kommission Aus-, Fort- und Weiterbildung der DGRW: Prof. Dr. Corinna Bergelt, PD Dr. Ruth Deck, Prof. Dr. Gert Krischak, Prof. Dr. Wilfried Mau (Sprecher), Prof. Dr. Matthias Morfeld, Prof. Dr. Susanne R. Schwarzkopf (PMU Salzburg), Prof. Dr. Karla Spyra, Susanne Walter + Mitglieder der Kommission Aus-, Fort- und Weiterbildung der DGPMR: Prof. Dr. Christoph Gutenbrunner, Prof. Dr. Uwe Lange, Dr. Max Liebl (Sprecher), Prof. Dr. Wilfried Mau, Dr. Anett Reißhauer, Prof. Dr. Susanne R. Schwarzkopf (PMU Salzburg), Prof. Dr. Ulrich C. Smolenski, Dr. Andreas Winkelmann

Dieser Artikel wird in den Zeitschriften „Die Rehabilitation“ und „Physikalische Medizin, Rehabilitationsmedizin, Kurortmedizin“ veröffentlicht.
} 


\section{ZUSAMMENFASSUNG}

Seit der ersten Publikation der Lernziele für den Querschnittsbereich „Rehabilitation, Physikalische Medizin, Naturheilverfahren (RPMN)“ im Medizinstudium im Jahr 2004 ist auf Grund heterogener Lehrangebote an den Fakultäten sowie neuer Impulse v. a. aus dem „Nationalen Kompetenzbasierten Lernzielkatalog Medizin (NKLM)“ und dem „Masterplan Medizinstudium 2020“ eine Revision sinnvoll. Deshalb haben die Deutsche Gesellschaft für Rehabilitationswissenschaften e. V. und die Deutsche Gesellschaft für Physikalische Medizin und Rehabilitation e. V. in einem strukturierten Konsensprozess nach der DELPHI-Methodik die Lernziele reduziert und übersichtlicher gegliedert. Besonders relevante Lernziele werden hervorgehoben. Alle Lernziele werden der kognitiven und Methodenkompetenz (Level 1 „Kennen und Können“) bzw. der Handlungskompetenz (Level 2 „Beherrschen“) zugeordnet. Auf die Lernziele im weniger detaillierten NKLM wird konkret verwiesen. Mit diesem revidierten Lernzielkatalog wird ein Beitrag zur Weiterentwicklung der kompetenzbasierten und in Kernbereichen homogeneren humanmedizinischen Lehre zu RPMN an den Fakultäten geleistet.

\section{ABSTRACT}

Since the first publication of learning objectives for the interdisciplinary subject "Rehabilitation, Physical Medicine, Naturopathic Treatment" in undergraduate medical education in 2004 a revision is reasonable due to heterogenous teaching programmes in the faculties and the introduction of the National Competence Based Catalogue of Learning Objectives in Medicine as well as the "Masterplan Medical Education 2020". Therefore the German Society of Rehabilitation Science and the German Society of Physical Medicine and Rehabilitation started a structured consensus process using the DELPHI-method to reduce the learning objectives and arrange them more clearly. Objectives of particular significance are emphasised. All learning objectives are assigned to the cognitive and methodological level 1 or to the action level 2. The learning objectives refer to the less detailed National Competence Based Catalogue of Learning Objectives in Medicine. The revised learning objectives will contribute to further progress in competence based and more homogenous medical teaching in core objectives of Rehabilitation, Physical Medicine, and Naturopathic Treatment in the faculties.

\section{Curriculare Lehre nach der 9. Revision der Ärztlichen Approbationsordnung}

Im Jahr 2003 wurde die Rehabilitation erstmals durch die 9. Revision der Ärztlichen Approbationsordnung (ÄAppO) im Medizinstudium curricular verankert und dem 12. Querschnittsbereich „Rehabilitation, Physikalische Medizin, Naturheilverfahren“ (RPMN) zugeordnet [1]. Um die deutschen Medizinischen Fakultäten bei der Umsetzung des damit verbundenen Lehrauftrags zu unterstützen, haben die Deutsche Gesellschaft für Rehabilitationswissenschaften e. V. (DGRW) und die Deutsche Gesellschaft für Physikalische Medizin und Rehabilitation e. V. (DGPMR) im Jahr 2004 einen gemeinsamen Lernzielkatalog empfohlen [2]. Darüber hinaus hat die DGRW in den Folgejahren grundsätzliche Erfordernisse der rehabilitationsbezogenen Lehre [3] und Lehr-/Prüfungsmethoden zusammengestellt [4-6] einschließlich der kostenfreien Bereitstellung von qualitätsgesicherten Multiple-Choice-Fragen für die Lehrbeauftragten an allen medizinischen Fakultäten [7]. Damit war die Hoffnung auf eine Angleichung der zentralen Lerninhalte zu RPMN im Studium der Humanmedizin in Deutschland verbunden, die nicht im Widerspruch zu standortspezifischen Profilbildungen im Detail steht. Bei mehreren Fragebogenerhebungen zu Strukturen und curricularen Inhalten an den Medizinischen Fakultäten in den letzten 12 Jahren [8-13] wurden zu jedem Befragungszeitpunkt Differenzen zwischen den einzelnen Standorten festgestellt. So wurde die Lehre zu RPMN an den meisten Fakultäten von Kliniken bzw. Instituten koordiniert, deren Name keinen direkten Zusammenhang mit der Bezeichnung des Querschnittsbereiches erkennen ließ. Bei der letzten Befragung der Fakultäten im Jahr 2015 [12] gab nur die Hälfte der Medizinischen Fakultäten Lehr-/Forschungsbereiche zu mind. einem der 3 RPMN-Teilbereiche an, was mit unterschiedlicher Durchführung der Lehre verbunden ist. Auch mehr als 12 Jahre nach Implementierung des Querschnittsbereichs RPMN und Publikation der gemeinsamen Empfehlung zu den entsprechenden Lernzielen wird die Lehre inhaltlich und didaktisch unterschiedlich gestaltet. Das betrifft gerade auch zentrale Bereiche, wie Zielstellung, behandelte Indikations- und Themenbereiche sowie Art und Umfang der Lehrveranstaltungen. Vorlesungen stehen im Medizinstudium i.d.R. immer noch weit im Vordergrund, obwohl sie passive, rezeptive Lernformen anregen, die im Sinne der Lernpyramide zu einem geringeren Lernerfolg und Kompetenzerwerb führen als z. B. die eigenständige Erarbeitung von Inhalten und die unmittelbare Anwendung des Gelernten unter Einsatz moderner didaktischer Konzepte und Methoden [4]. Trotz dieser Erkenntnis finden strukturierte Fallbeispiele, problemorientiertes Lernen und E-Learning aktuell selten Anwendung [12].

\section{Akzente des Nationalen Kompetenz- basierten Lernzielkatalogs Medizin}

Zur Standardisierung der Curricula für das Medizinstudium ist aktuell der „Nationale Kompetenzbasierte Lernzielkatalog Medizin (NKLM)“ besonders relevant, der durch den Medizinischen Fakultätentag der Bundesrepublik Deutschland e.V. und die Gesellschaft für Medizinische Ausbildung erstellt wurde $[14,15]$. Der Katalog zielt darauf ab, ein „Absolventenprofil von Ärztinnen/Ärzten im Sinne eines Kerncurriculums für das Studium der Medizin“ zu beschreiben. Damit bietet er auch eine Orientierung für die einheitlichere und fokussiertere Ausgestaltung der Lehre zu RPMN. Er versteht sich als Ergänzung für die Gegenstandskataloge des Instituts für Medizinische und Pharmazeutische Prüfungsfragen (IMPP) für die schriftlichen Teile der Staatsexamina und nimmt Bezug auf $\S 1$ Abs. 1 der ÄAppO, nach der im Medizinstudium „grundlegende 
Kenntnisse, Fähigkeiten und Fertigkeiten in allen Fächern (...), die für eine umfassende Gesundheitsversorgung der Bevölkerung erforderlich sind“, vermittelt werden sollen [14,15]. Dabei soll das reine Faktenwissen durch die Förderung und Einübung von Fähigkeiten, Fertigkeiten und professionellen Haltungen ergänzt werden. Das soll dazu führen, dass sich die ärztliche Versorgung in jedem Einzelfall verbessert, v. a. auch im Hinblick auf patientenzentrierte, evidenzbasierte Entscheidungen und die Ausschöpfung des gesamten Spektrums präventiver, kurativer, rehabilitativer und palliativer Leistungen.

\section{Revisionsprozess des Lernzielkatalogs „Rehabilitation, Physikalische Medizin, Naturheilverfahren“ (RPMN)}

Mit der Weiterentwicklung der Medizin und didaktischer Konzepte sowie dem nun verfügbaren NKLM ergab sich die Notwendigkeit, den Lernzielkatalog von 2004 zu überarbeiten. Dazu haben die beiden Kommissionen für Aus-, Fort- und Weiterbildung der DGRW und der DGPMR gemeinsam eine grundlegende Revision vorgenommen. Sie umfasst sowohl die Integration von Kompetenzen der Studierenden, als auch eine Reduktion der Inhalte mit Hervorhebung besonders relevanter Aspekte und Verlagerung von Details auf die Beispielebene. Darüber hinaus beinhaltet sie zahlreiche Querverweise zwischen den einzelnen Bereichen des revidierten Lernzielkatalogs sowie auf den NKLM. Dazu wurde ein strukturierter Konsensprozess nach der DELPHI-Methodik [16] durchgeführt. In einem Kick-off-Workshop am 17.10.2016 in Halle (Saale) wurden relevante Materialien gesichtet und das grundsätzliche Vorgehen mit initialer Arbeitsteilung und anschließender Zusammenführung der Ergebnisse abgestimmt. Auf Basis der Fassung des Lernzielkatalogs von 2004, des NKLM sowie aktueller Daten und Entwicklungen hat die DGRW-Kommission zunächst den Teil Rehabilitation und die DGPMR-Kommission die Teile Physikalische Medizin und Naturheilverfahren des früheren Lernzielkatalogs entsprechend dem oben genannten Vorgehen mit 2 Abstimmungen per E-Mail überarbeitet. Die Ergebnisse wurden von WM, ML und CG zusammengeführt und am 11.07.2017 in Hannover in einer gemeinsamen Sitzung der beiden Kommissionen konsentiert. Das Ergebnis wurde anschließend von den beiden Vorständen der DGRW und der DGPMR verabschiedet.

\section{Überlegungen zur Umsetzung des kom- petenzbasierten Lernzielkatalogs RPMN}

Auch für die Anwendung des revidierten Lernzielkatalogs RPMN müssen Überlegungen angestellt werden, wie in der tatsächlichen Lehrpraxis der Unterricht so gestaltet werden kann, dass er nicht nur zu einem reinen Wissenszuwachs führt, sondern die zukünftigen Ärztinnen/Ärzte dazu befähigt, die Inhalte des Querschnittsbereichs in der klinischen Praxis umzusetzen und anzuwenden. Hierfür wird es notwendig sein, neben dem Grundverständnis für die Mechanismen, Potenziale und leistungsrechtlichen Voraussetzungen der einzelnen therapeutischen Verfahren und komplexen Rehabilitationsleistungen, deren Indikationsstellung bzw. Anwen- dung bei bestimmten Funktionsstörungen und Krankheitsbildern zu üben und auf den Einzelfall anzuwenden. Dabei wird die Trennung zwischen den einzelnen durch die ÄAppO vorgegebenen Teilbereichen (Rehabilitation, Physikalische Medizin, Naturheilverfahren) zwangsläufig aufgehoben werden müssen, damit die Ärztin/ der Arzt in die Lage versetzt wird, adäquate klinische Entscheidungen zu treffen ( $\triangleright$ Tab. 1). Dies steht nicht im Widerspruch dazu, dass aus didaktischen Erwägungen die einzelnen Teilbereiche für die Wissensvermittlung systematisch untergliedert werden ( $\triangleright$ Tab. 2). Die in $>$ Tab. 1 dargestellten Kompetenzen sind im Querschnittsbereich RPMN von besonderer Bedeutung und sollen somit in die studentische Lehre im Medizinstudium integriert werden.

Darüber hinaus stellt sich die Frage, inwieweit der Querschnittsbereich RPMN spezifische Elemente für die 3 großen Abschnitte des NKLM (Rollen der Ärztin/des Arztes; Medizinisches Wissen, klinische Fähigkeiten und professionelle Haltungen; Patientenzentrierte Gesundheitsversorgung) beitragen kann und muss. Angesichts der Tatsache, dass in relativ großem Umfang Therapien durch andere Berufsgruppen durchgeführt werden, in vielen Fällen Patienten mit chronischen Erkrankungen, Multimorbidität, komplexen (psychosozialen) Problemlagen sowie gravierenden Behinderungen behandelt werden und die Ziele wesentlich über die Therapie von Krankheiten (im Sinne der Integration in das soziale Leben) hinausgehen, scheint dies tatsächlich der Fall zu sein. Einige Aspekte diesbezüglich sind:

- Rollen der Ärztin/des Arztes: Der Abschnitt 1 des NKLM bezieht sich in seiner Darstellung der übergeordneten Kompetenzen auf das CanMEDS-Modell, in dem 7 Arztrollen bzw. Teilkompetenzen differenziert werden: Die integrative Rolle als Medical Expert (im NKLM: Medizinische/-r Experte/-in) sowie die Rollen Communicator (Kommunikator/-in), Collaborator (Mitglied eines Teams), Leader

(Verantwortungsträger/-in \& Manager/-in), Health Advocate (Gesundheitsberater/-in \& - fürsprecher/-in), Scholar (Gelehrte/-r) und Professional (Professionell Handelnde/-r) [17]. Hier kann der Querschnittsbereich RPMN für die Vermittlung verschiedener Kompetenzrollen einen Beitrag leisten. Dies beinhaltet einerseits die Überwindung natürlicher Unsicherheiten in der Kommunikation mit Menschen mit Behinderungen, der speziellen Rolle des Arztes/der Ärztin in der Betreuung von Menschen mit schweren und chronischen Behinderungen und der individuellen partizipativen Entscheidungsfindung bei der Festlegung von Rehabilitationszielen. Die Rollen als Communicator, aber auch als Health Advocate (im Sinne der Fürsprecherin/des Fürsprechers des Patienten) und Professional mit einer ethisch hochstehenden ärztlichen Haltung sind hier besonders relevant. Sie setzen Kenntnisse zu Krankheitsfolgen und Kontextfaktoren der Betroffenen entsprechend bio-psycho-sozialen Modellen wie der Internationalen Klassifikation der Funktionsfähigkeit, Behinderung und Gesundheit (ICF) sowie der Behandlungsmöglichkeiten im Spektrum der RPMN voraus, die in der Rolle des Medical Experts zu erwerben sind. Sowohl an Betroffene als auch an verschiedene Berufsgruppen in der Rehabilitation sind diese Kenntnisse in der Rolle der/des Gelehrten (Scholar) z. B. in Patientenschulungen bzw. in der Aus- und Fortbildung weiterzugeben. 
- Tab. 1 Besonders relevante Kompetenzen im Querschnittsbereich „Rehabilitation, Physikalische Medizin, Naturheilverfahren“.

\begin{tabular}{|c|c|c|c|c|}
\hline Nr. & Bereich & Thema & $\begin{array}{l}\text { Kompetenzlevel 1: } \\
\text { Kennen und Können }\end{array}$ & $\begin{array}{l}\text { Kompetenzlevel 2: } \\
\text { Beherrschen }\end{array}$ \\
\hline \multirow[t]{2}{*}{1.} & $\begin{array}{l}\text { Theoretische } \\
\text { Grundlagen }\end{array}$ & Wirkungsmechanismen & $\begin{array}{l}\text { Reiz-Reaktions-Prinzip, funktionelle Adaptation, } \\
\text { Lernen und Verhaltensänderung } \\
\text { Auslösung von Adaptationen und ihre } \\
\text { Auswirkungen auf Funktionen }\end{array}$ & \\
\hline & & $\begin{array}{l}\text { Theoriemodelle von Behinderung } \\
\text { und Rehabilitation }\end{array}$ & ICF-Modell der WHO & $\begin{array}{l}\text { Beschreibung von Problemen } \\
\text { und Ressourcen im ICF-Modell }\end{array}$ \\
\hline \multirow[t]{2}{*}{2.} & $\begin{array}{l}\text { Diagnostik und } \\
\text { Assessment }\end{array}$ & Funktions- und Leistungsdiagnostik & Funktions- und Leistungstests & $\begin{array}{l}\text { Klinische Untersuchungstechni- } \\
\text { ken, ausgewählte Leistungstests }\end{array}$ \\
\hline & & Rehabilitative Assessments & $\begin{array}{l}\text { Assessmentfragebögen und Checklisten, } \\
\text { klinische Assessments } \\
\text { Aussagekraft der Assessmentinstrumente }\end{array}$ & $\begin{array}{l}\text { Auswertung und Interpretation } \\
\text { ausgewählter Assessmentinstru- } \\
\text { mente }\end{array}$ \\
\hline \multirow[t]{7}{*}{3.} & Interventionen & $\begin{array}{l}\text { Physiotherapie, Ergotherapie, } \\
\text { Stimm- und Sprachtherapie }\end{array}$ & $\begin{array}{l}\text { Grundlagen, Indikationen und Kontra- } \\
\text { indikationen } \\
\text { Berufsverständnis der Spezialtherapeuten }\end{array}$ & Indikationsstellung \\
\hline & & Weitere Physikalische Therapien & $\begin{array}{l}\text { Wirkungen, Indikationen und Kontra- } \\
\text { indikationen }\end{array}$ & Indikationsstellung \\
\hline & & Heil- und Hilfsmittelverordnung & $\begin{array}{l}\text { Heil- und Hilfsmittelrezepte, Heil- und } \\
\text { Hilfsmittelkatalog }\end{array}$ & Rezeptieren \\
\hline & & $\begin{array}{l}\text { Physikalische Therapien in Behand- } \\
\text { lungsstrategien }\end{array}$ & $\begin{array}{l}\text { Differenzierter Einsatz und Wertigkeit } \\
\text { physikalischer Therapien in Behandlungs- } \\
\text { konzepten }\end{array}$ & $\begin{array}{l}\text { Einsatz als alleinige, prioritäre } \\
\text { oder additive physikalische } \\
\text { Therapien }\end{array}$ \\
\hline & & $\begin{array}{l}\text { Medikamentöse Therapien in der } \\
\text { Physikalischen Medizin und } \\
\text { Rehabilitation }\end{array}$ & $\begin{array}{l}\text { Strategien der Pharmakotherapie in der } \\
\text { Rehabilitation } \\
\text { Wechselwirkungen mit Physikalischen und } \\
\text { Psychotherapien }\end{array}$ & Verordnung von Medikamenten \\
\hline & & Naturheilverfahren & $\begin{array}{l}\text { Einschätzung der evidenzbasierten Wirksam- } \\
\text { keit und des Stellenwerts von Naturheilverfah- } \\
\text { ren im Therapiespektrum }\end{array}$ & \\
\hline & & $\begin{array}{l}\text { Edukative und psychosoziale } \\
\text { Interventionen (z. B. körperorientier- } \\
\text { te psychotherapeutische Verfahren, } \\
\text { Ernährungsberatung und rehabilitati- } \\
\text { ve Sozialarbeit) }\end{array}$ & $\begin{array}{l}\text { Grundkenntnisse der Wirkungsweise } \\
\text { Integration in kognitiv-behaviorale Strategien }\end{array}$ & Indikationsstellung \\
\hline \multirow[t]{3}{*}{4.} & $\begin{array}{l}\text { Komplexe } \\
\text { Rehabilitations- } \\
\text { programme }\end{array}$ & $\begin{array}{l}\text { Krankheitsspezifische und krank- } \\
\text { heitsübergreifende Rehabilitations- } \\
\text { leistungen }\end{array}$ & $\begin{array}{l}\text { Ziele und Organisation der einzelnen } \\
\text { Rehabilitationsformen } \\
\text { Multiprofessionelles und interdisziplinäres } \\
\text { Rehabilitationsteam } \\
\text { Frührehabilitation im Krankenhaus, postakute } \\
\text { und Anschlussrehabilitation, ambulante, } \\
\text { stationäre und aufsuchende Rehabilitation bei } \\
\text { chronischen Erkrankungen, rehabilitative } \\
\text { Versorgung durch niedergelassene Ärztinnen } \\
\text { und Ärzte }\end{array}$ & $\begin{array}{l}\text { Erstellen von Rehabilitations- } \\
\text { plänen } \\
\text { (an ausgewählten Beispielen) }\end{array}$ \\
\hline & & Andere Rehabilitationsangebote & $\begin{array}{l}\text { schulisch-pädagogische, berufliche und soziale } \\
\text { Rehabilitation (Grundkenntnisse) }\end{array}$ & \\
\hline & & $\begin{array}{l}\text { Veranlassung und Einleitung von } \\
\text { Rehabilitationsleistungen }\end{array}$ & relevante leistungsrechtliche Bestimmungen & $\begin{array}{l}\text { Veranlassung von Rehabilita- } \\
\text { tionsleistungen } \\
\text { (an ausgewäh/ten Beispielen) }\end{array}$ \\
\hline 5. & $\begin{array}{l}\text { Ausgewählte } \\
\text { Krankheitsbilder } \\
\text { und Funktions- } \\
\text { störungen }\end{array}$ & $\begin{array}{l}\text { Akuter und chronischer Schmerz, } \\
\text { Erkrankungen des Bewegungs- } \\
\text { systems, des Nervensystems, des } \\
\text { Herz-Kreislaufsystems und der } \\
\text { Atmung, onkologische Erkrankun- } \\
\text { gen, psychosomatische Störungen, } \\
\text { psychosoziale Problemlagen }\end{array}$ & $\begin{array}{l}\text { Therapie- und Rehabilitationsbedarf sowie } \\
\text { Strategien bei akuten und chronischen } \\
\text { Krankheitsbilder und Funktionsstörungen }\end{array}$ & $\begin{array}{l}\text { Diagnostik, Assessment und } \\
\text { Erstellung von Therapie- und } \\
\text { Rehabilitationsplänen } \\
\text { (an ausgewählten Fällen) }\end{array}$ \\
\hline
\end{tabular}




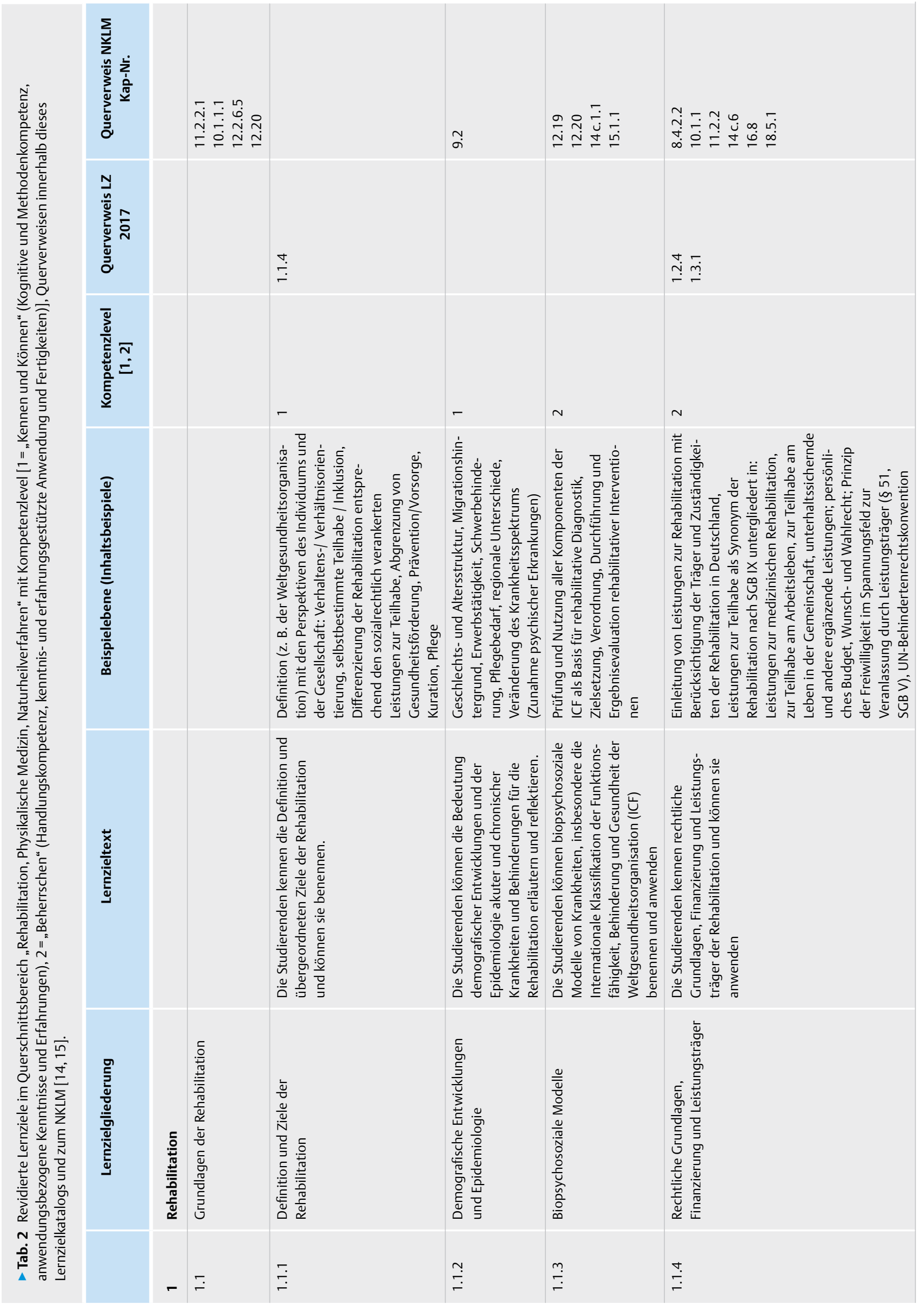



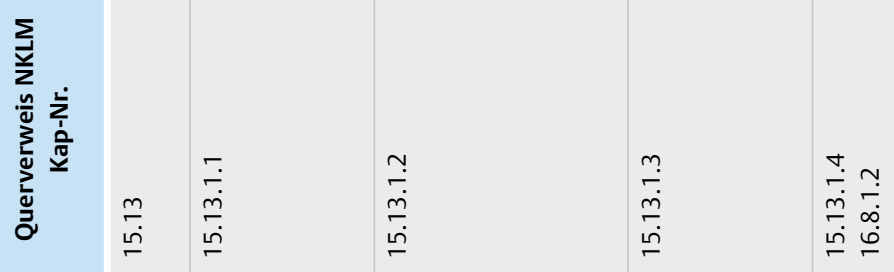

ำ
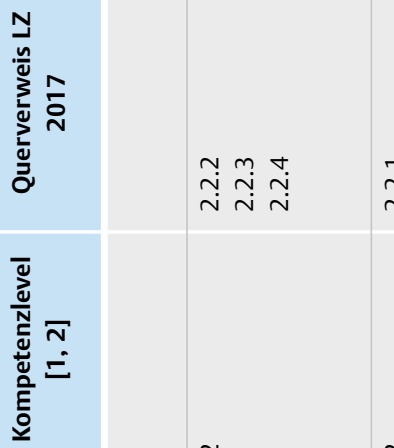

i

i

$\stackrel{+}{=}$

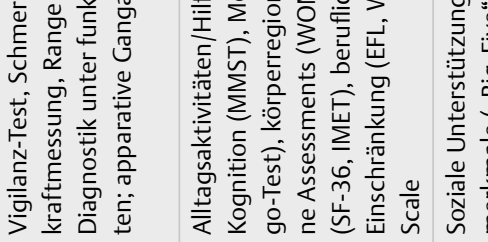
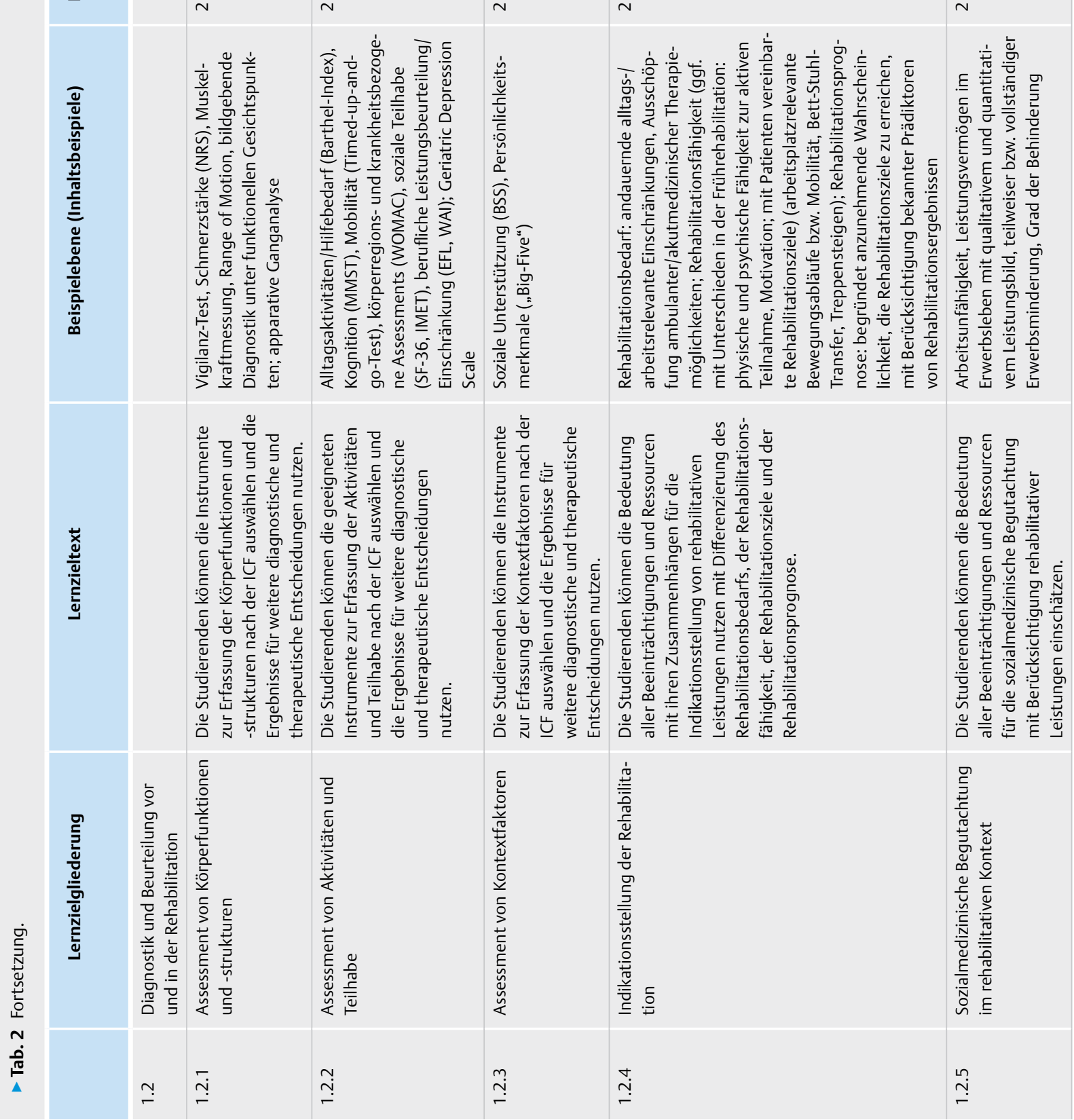


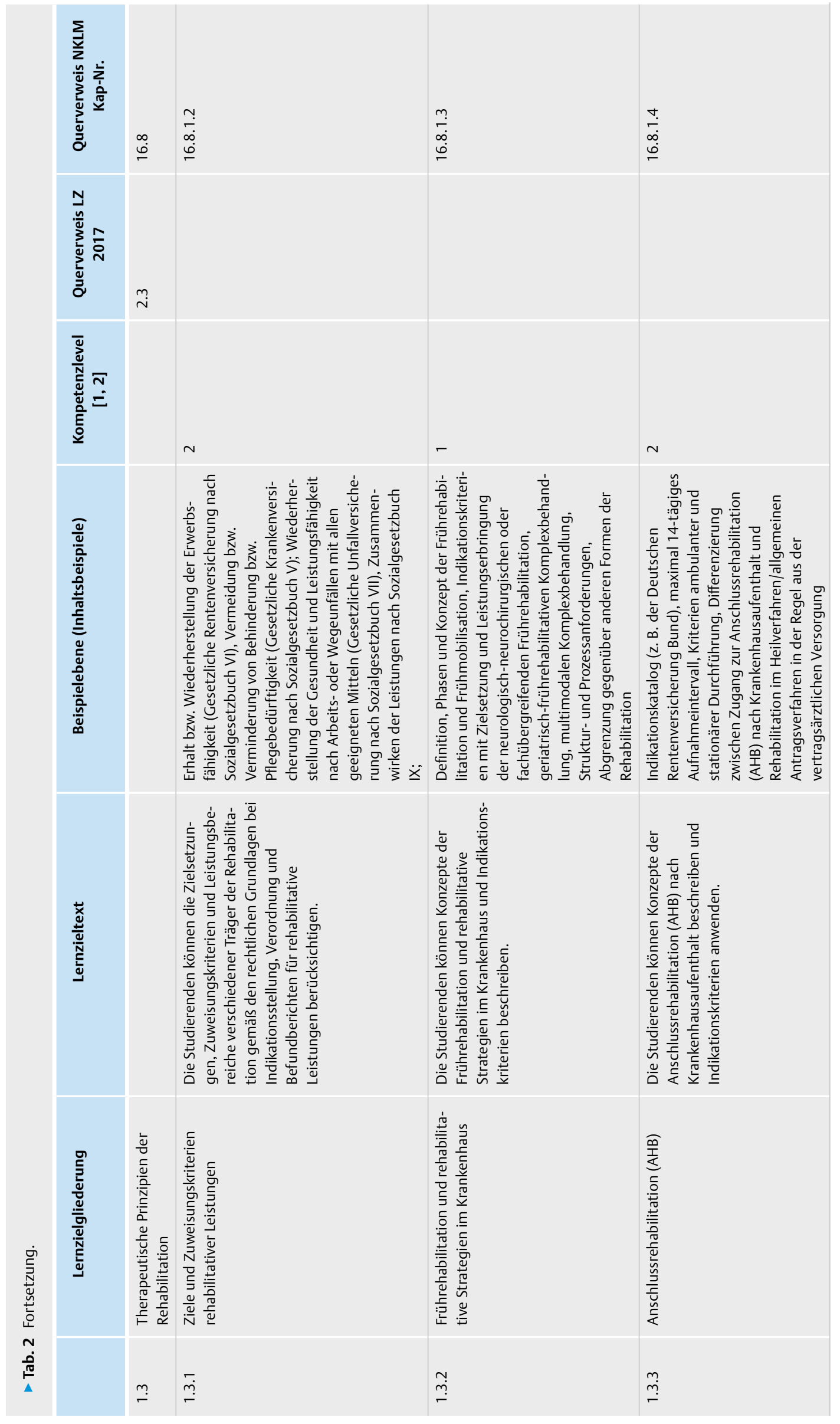



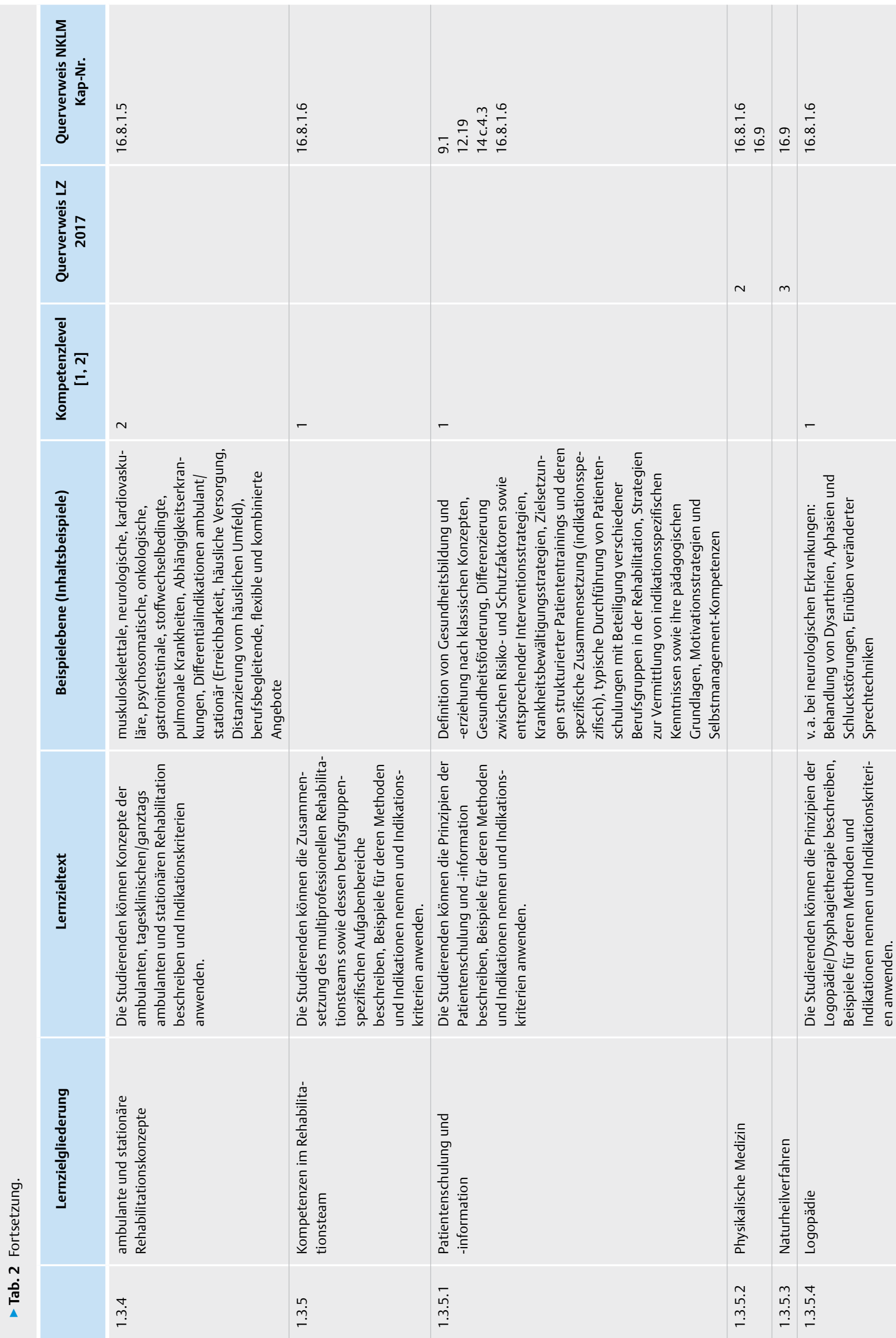


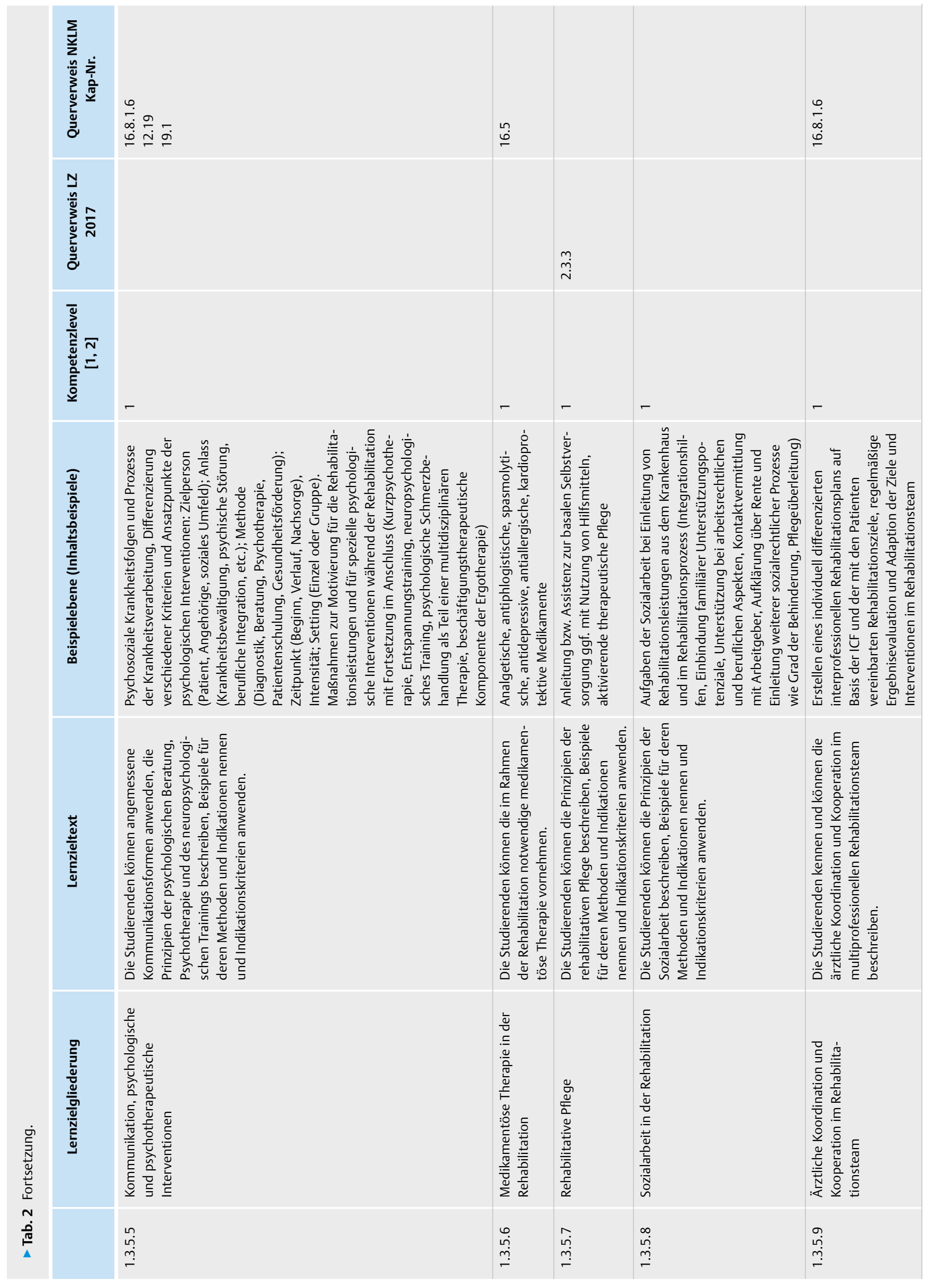



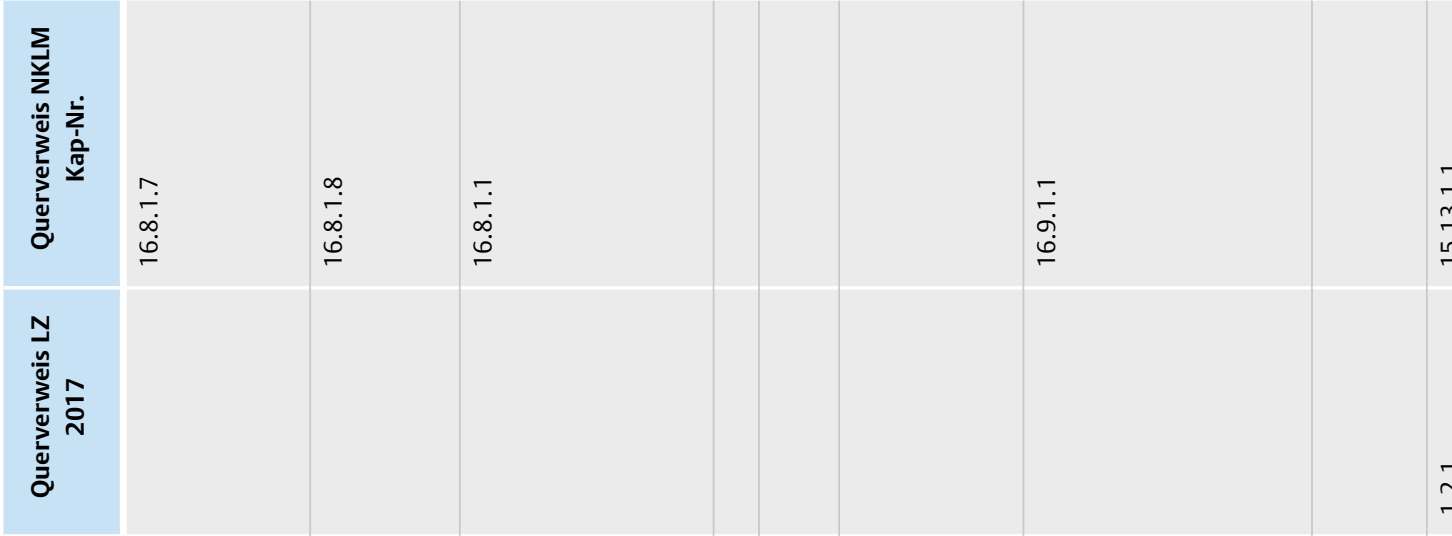

ฮำำ
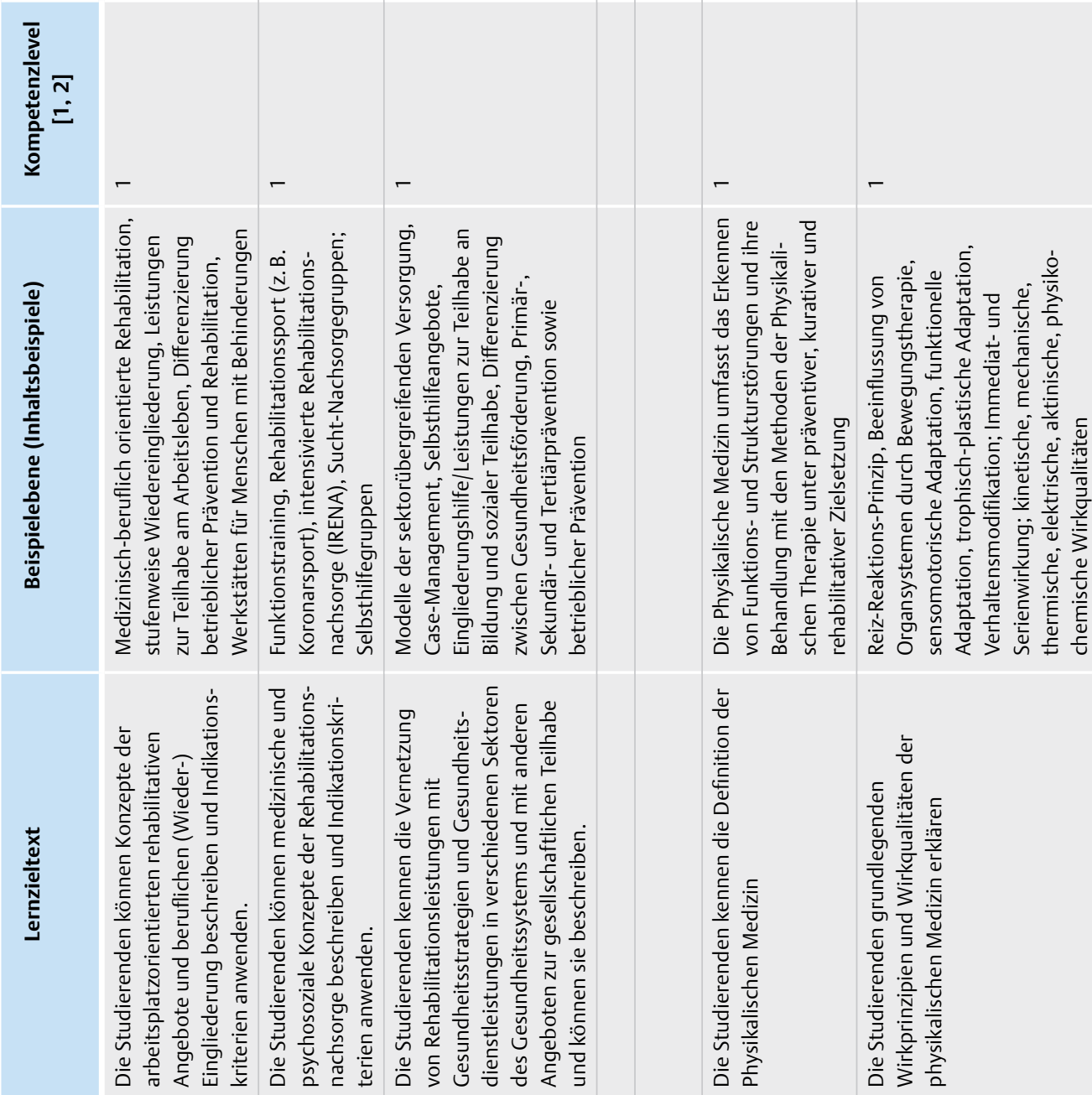

N
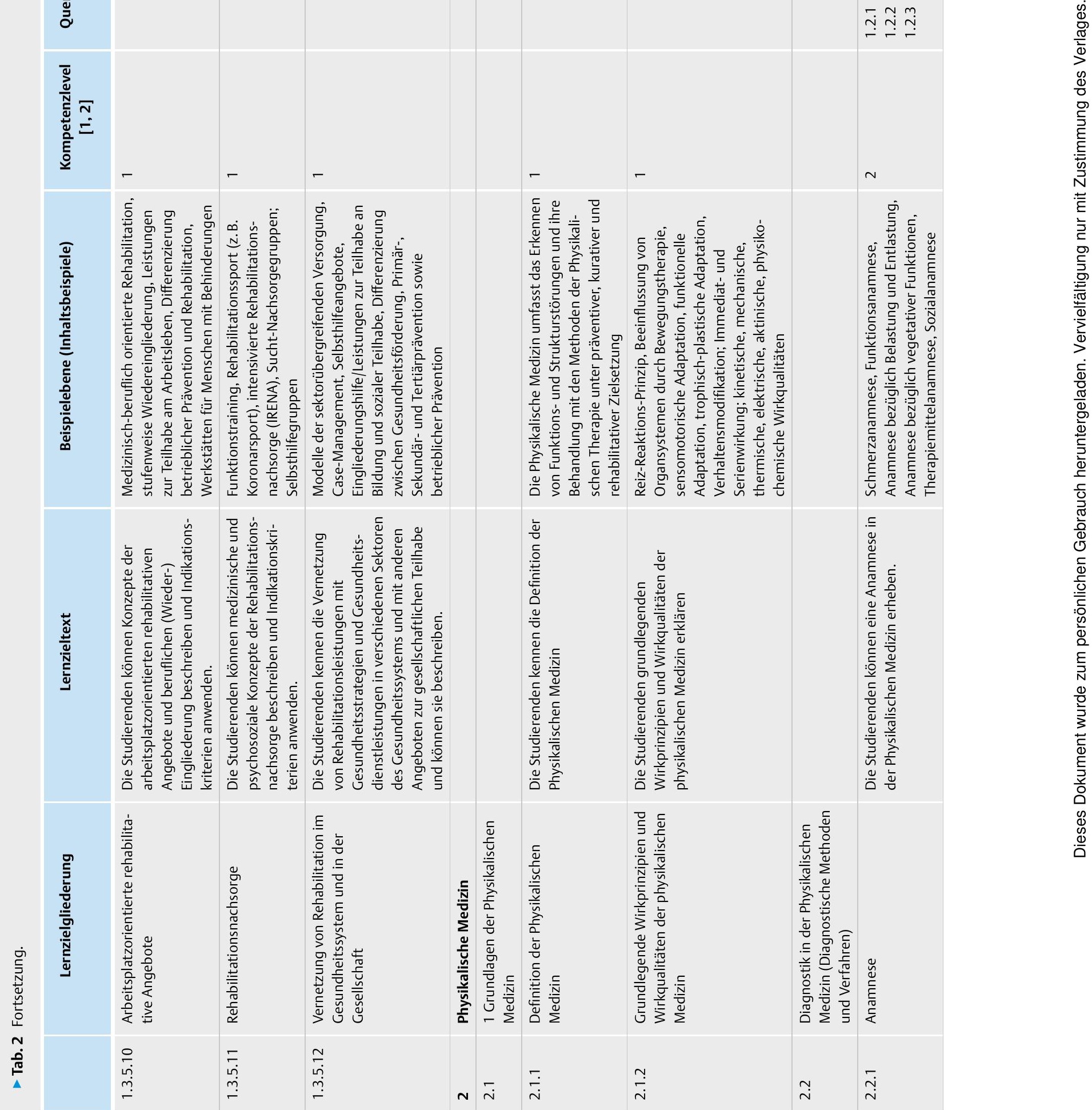


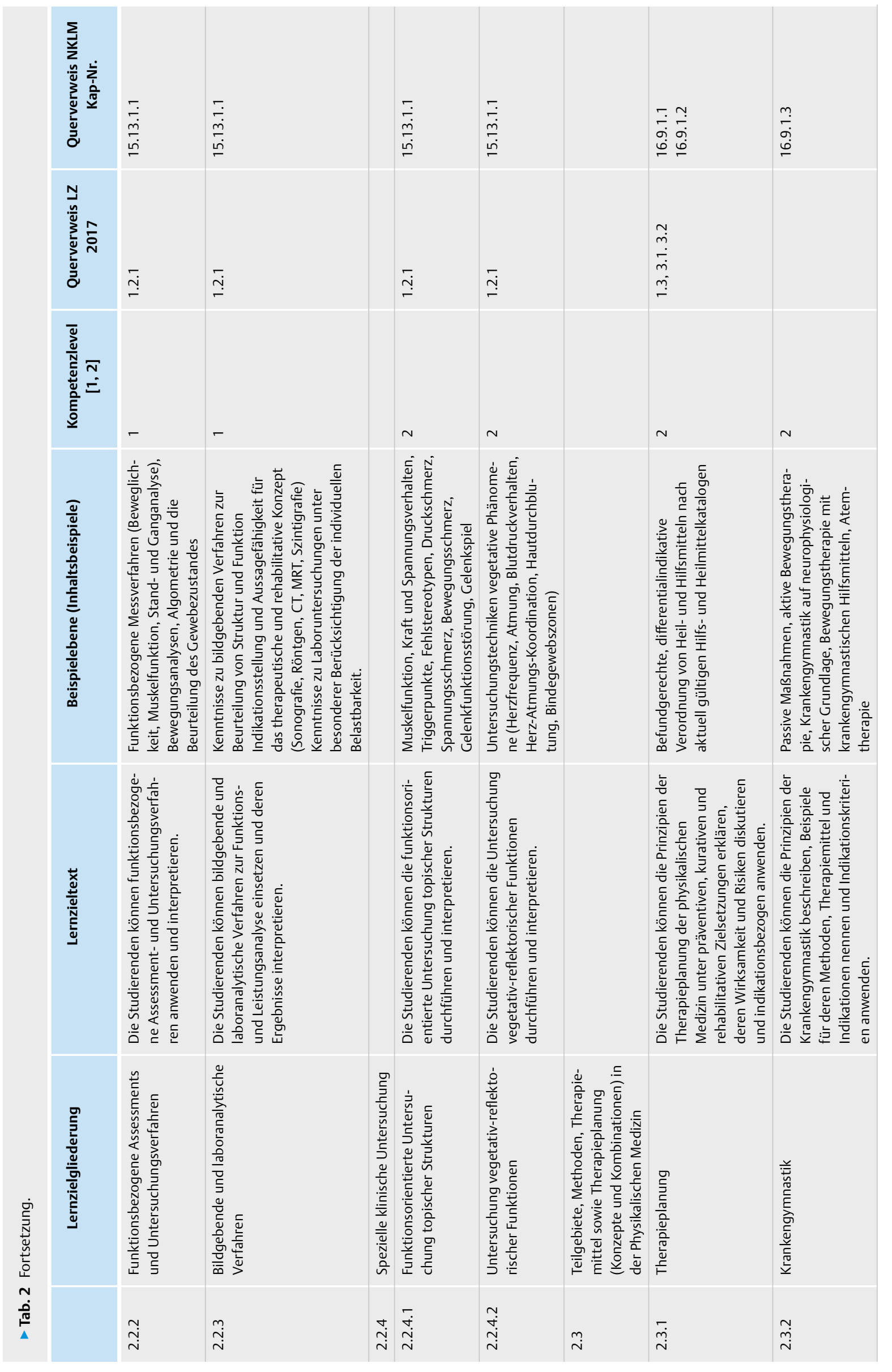



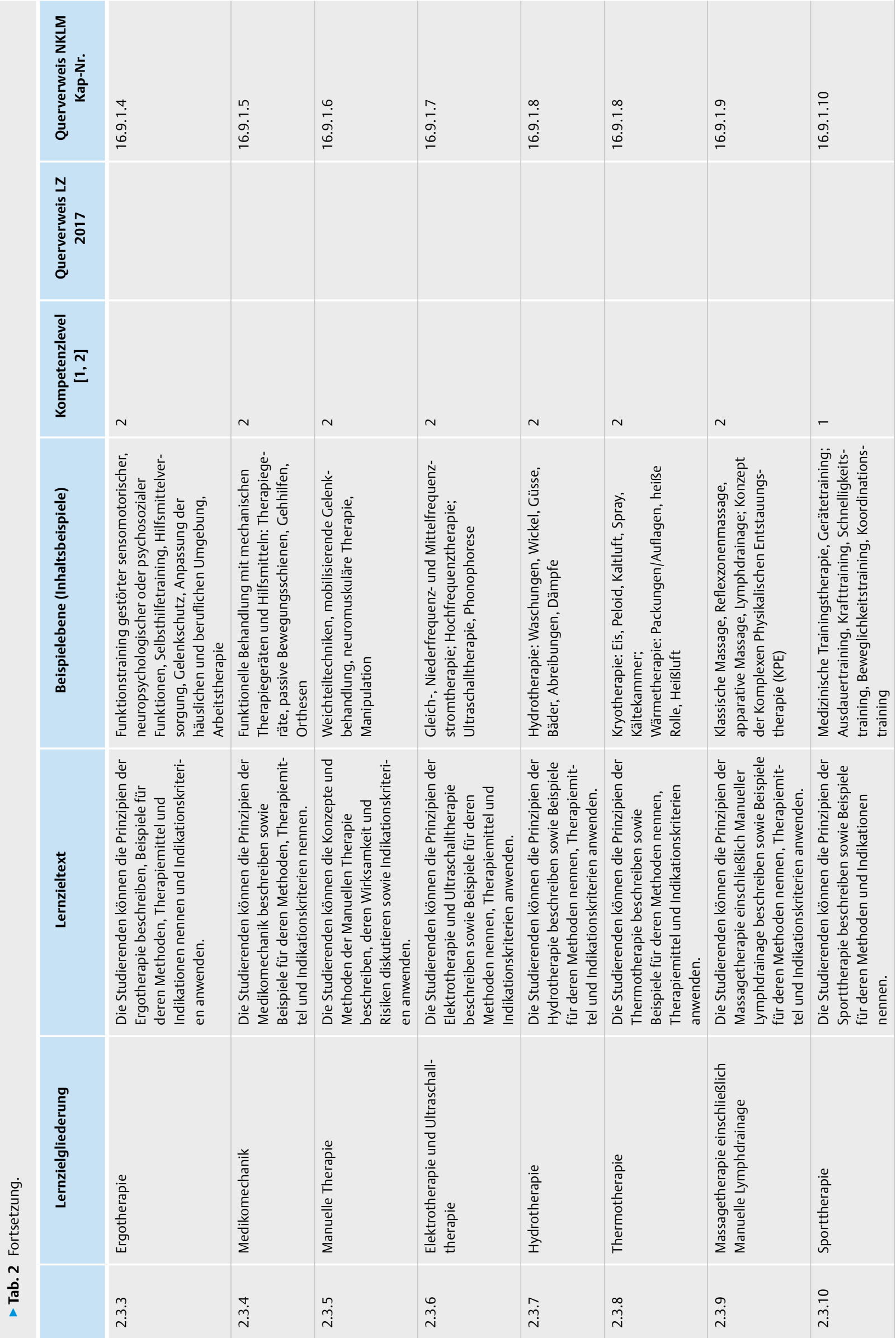


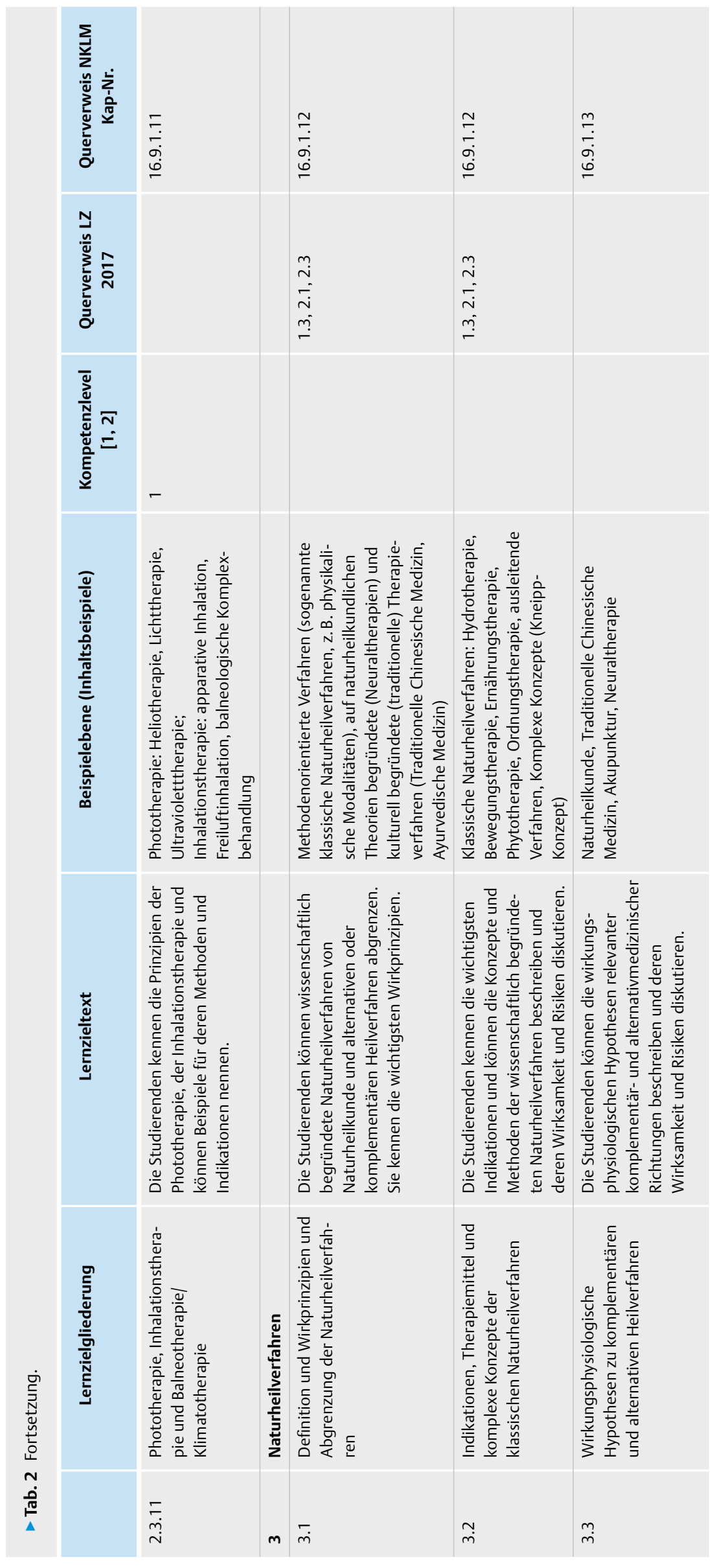


Anderseits ist das Erlernen der Arztrolle im multiprofessionellen Behandlungs- bzw. Rehabilitationsteam (Collaborator) essenziell, was auch ein Verständnis von berufsgruppenspezifischen Denk- und Ausdruckweisen beinhalten muss. Ferner ist die Kenntnis der wesentlichen rechtlichen Grundlagen der Gesundheits- und Krankenversorgung, v. a. der Rehabilitation, essentiell für eine Verbesserung der Gesundheitsversorgung chronisch kranker und behinderter Personen. Hier können im Fach RPMN, neben einer verschiedene Gesundheitsfachberufe betreffenden übergreifenden Methodenkompetenz der Absolventen, insbesondere die Rollen als Verantwortungsträger/-in und Manager/-in (Leader) differenziert und gestärkt werden.

- Medizinisches Wissen, klinische Fähigkeiten und professionelle Haltungen: Das Medizinische Wissen muss - wie in anderen Fächern - zunächst die Wirkungsmechanismen, Indikationen und Kontraindikationen der Therapiemittel beinhalten. Spezifisch für den Querschnittsbereich RPMN sind die Zuordnung der Therapien zu den besonderen Funktionsstörungen im Einzelfall und die Zuweisung zu Therapeuten/-innen sowie in spezialisierte Rehabilitationseinrichtungen. Was die professionelle Haltung angeht, so ist insbesondere für die bereits erwähnte partizipative Entscheidungsfindung sowie die Teamkooperation in der Leistungserbringung zu sensibilisieren.

- Patientenzentrierte Gesundheitsversorgung: Was die konkreten Konsultationsanlässe und Erkrankungen angeht, die in der Versorgung entsprechend dem Spektrum des Querschnittsbereichs relevant sind, so sollte die Anwendung physikalischer und rehabilitativer Interventionen bei häufigen Krankheiten (z. B. muskuloskeletale und degenerative Erkrankungen, Gliedmaßenamputation, Querschnittläsionen, neurologische Erkrankungen), Funktionsstörungen (z. B. Schmerzen, Spastizität) und Behinderungen (z. B. Aktivitäten des täglichen Lebens, Einschränkungen der beruflichen Leistungsfähigkeit) einschließlich der Teilhabe (z. B. Familie, Beruf und Freizeit) und Berücksichtigung von relevanten Kontextfaktoren (z. B. Arbeitsumfeld, Familie, Merkmale der Person) in der Lehre als spezifischer Lehrinhalt integriert werden (mind. exemplarisch). Hier erscheint es notwendig, Pathways (einschl. Kombinationen und Priorisierungen von Therapien) für bestimmte Krankheitsbilder und Funktionsstörungen zu erarbeiten und auf konkrete Patientenfälle anzuwenden.

\section{Ausblick}

Diese Empfehlungen sollten in geeigneter Weise in die Lehre im Querschnittsbereich RPMN einfließen und Bestandteil der Curricula einschließlich Prüfungen werden. Zusammen mit dem NKLM werden sie z. B. beim Aufbau einer Datenbank in Form eines WikiSystems zu Lehrmaterialien mit zugeordneten Lernzielen berücksichtigt [18], die Dozenten für die Durchführung interdisziplinärer rehabilitationsbezogener Lehre zur Verfügung gestellt werden. Die revidierten Lernziele werden den aktuellen Forderungen im „Masterplan Medizinstudium 2020“ gerecht, die bestehende Lehrinhalte mit Konzentration auf wesentliche Lernziele und -inhalte weiterzuentwickeln und umzustrukturieren [19].
Auch wenn mit diesem Lernzielkatalog primär ein Beitrag zur Weiterentwicklung der kompetenzbasierten humanmedizinischen Lehre zu RPMN geleistet werden soll, können sich daraus auch für die Curricula anderer Berufsgruppen im interprofessionellen Rehabilitationsteam [20] Anregungen ergeben.

\section{Danksagung}

Die Autoreninnen/Autoren danken Frau Stefanie Schmidt aus Halle für die Unterstützung bei der Erstellung der Lernzieltabellen.

Interessenkonflikt

Die Autoren geben an, dass kein Interessenkonflikt besteht.

Literatur

[1] Bundesministerium für Gesundheit. Approbationsordnung für Ärzte vom 27. Juni 2002 Bundesgesetzblatt Teil 1 2002; 44: 2405-2435

[2] Mau W, Gülich M, Gutenbrunner Ch et al Lernziele im Querschnittsbereich Rehabilitation, Physikalische Medizin und Naturheilverfahren nach der 9. Revision der Approbationsordnung für Ärzte. Gemeinsame Empfehlungen der Deutschen Gesellschaft für Rehabilitationswissenschaften und der Deutschen Gesellschaft für Physikalische Medizin und Rehabilitation. Rehabilitation 2004; 43: 337-347

[3] Mau W, Kawski S, Lay W. Erfordernisse der Ausbildung zur Rehabilitation in der humanmedizinischen Lehre: Positionspapier der Deutschen Gesellschaft für Rehabilitationswissenschaften (DGRW). Rehabilitation 2010; 49: 114-119

[4] Schwarzkopf SR, Morfeld M, Gülich M et al. Aktuelle Lehr-, Lern- und Prüfungsmethoden in der medizinischen Ausbildung und ihre Anwendungsmöglichkeiten für die Rehabilitation. Rehabilitation 2007; 46: 64-73

[5] Horn K, Gülich M, Lay W et al. Problemorientiertes Lernen (POL) in der Rehabilitation am Beispiel eines Patienten mit Spondylitis ankylosans. Rehabilitation 2007; 46: 164-174

[6] Morfeld M, Mau W, Jäckel WH, Koch U (Hrsg). Im Querschnitt: Rehabilitation, Physikalische Medizin und Naturheilverfahren. Ein fallorientiertes Lehrbuch. München: Elsevier, Urban \& Fischer; 2007

[7] Gutt S, Bergelt C, Faller $\mathrm{H}$ et al. Entwicklung eines Prüfungsfragenpools für die rehabilitationsbezogene Lehre im Studiengang Humanmedizin. Rehabilitation 2015; 54: 259-265

[8] Mau W, Kusak G. Umsetzung der neuen Approbationsordnung für Ärzte im Querschnittsbereich „Rehabilitation, Physikalische Medizin und Naturheilverfahren “ an den medizinischen Fakultäten in Deutschland. Rehabilitation 2005; 44: 129-133

[9] Kusak G, Gülich M, Lay W et al. Entwicklung der Lehre im Querschnittsbereich „Rehabilitation, Physikalische Medizin, Naturheilverfahren“ an den medizinischen Fakultäten 2004-2006/07. Rehabilitation 2008; 47: 2-7

[10] Gutt S, Uhlmann A, Faller H et al. Neue Entwicklungen bei E-Learning-, Prüfungs- und Evaluationsmodalitäten in der rehabilitationsbezogenen Lehre - Ergebnisse der DGRW Fakultätenbefragung 2010. DRV-Schriften 2011: 93: 189-191

[11] Mau W. Durchführung der Lehre im Querschnittsbereich „Rehabilitation, Physikalische Medizin, Naturheilverfahren " an den Medizinischen Fakultäten in Deutschland. Phys Med Rehab Kuror 2010; 20: 327-331 
[12] Schmidt S, Bergelt C, Deck R et al. Aktuelle Entwicklung der rehabilitationsbezogenen Lehre in den humanmedizinischen Studiengängen in Deutschland: Ergebnisse der DGRW-Fakultätenbefragung 2015. Rehabilitation 2017; 56: 47-54

[13] Stock-Schröer B, Huber R, Joos S, Klose P. Der Unterricht für Medizinstudierende in Rehabilitation, Physikalischer Medizin und Naturheilverfahren - eine deutschlandweite Bestandsaufnahme 10 Jahre nach Reform der ärztlichen Approbationsordnung. GMS J Medical Education 2017; 34: 8-15

[14] MFT - Medizinischer Fakultätentag der Bundesrepublik Deutschland e.V. Nationaler Kompetenzbasierter Lernzielkatalog Medizin, verfügbar unter: http://www.mft-online.de/lehre/nationaler-kompetenzbasierter-lernzielkatalog-medizin Zugriff vom 30.06.2017

[15] Fischer MR, Bauer D, Mohn K. Endlich fertig! Nationale Kompetenzbasierte Lernzielkataloge Medizin (NKLM) und Zahnmedizin (NKLZ) gehen in die Erprobung. GMS Z Med Ausbild 2015; 32: Doc 35

[16] Ammon U. Delphi-Befragung. In: Kühl S, Strodtholz P, Taffertshofer A (Hrsg). Handbuch Methoden der Organisationsforschung - Quantitative und Qualitative Methoden. Wiesbaden: VS Verlag für Sozialwissenschaften; 2009: 458-476
[17] Frank JR, Snell L, Sherbino J (Hrsg). CanMEDS 2015 Physician Competency Framework. Ottawa: Royal College of Physicians and Surgeons of Canada; 2015

[18] Schmidt S, Schwabe S, Wichmann D, Mau W. Entwicklung einer Datenbank (MediaWiki) für Dozenten interdisziplinärer rehabilitationsbezogener Lehre. In: Deutsche Rentenversicherung Bund $(\mathrm{Hg})$ : DRV-Schriften 2017; 11: 178-179

[19] Bundesministerium für Bildung und Forschung. „Masterplan Medizinstudium 2020“ beschlossen am 31. März 2017 in einer gemeinsamen Konferenz der Ministerinnen und Minister, Senatorinnen und Senatoren für Gesundheit von Bund und Ländern sowie den Bundestagsfraktionen von CDU/CSU und SPD“. Abrufbar unter: https://www.bmbf.de/de/masterplan-medizinstudium-2020-4024. html (zuletzt aufgerufen am 14.07.2017)

[20] Mau W, Bengel J, Pfeifer K. Rehabilitation in der Aus-, Fort- und Weiterbildung beteiligter Berufsgruppen. Bundesgesundheitsblatt Gesundheitsforschung - Gesundheitsschutz 2017; 60: 402-409 